\title{
Three
}

\section{STEADY STATE AND PLASMA COSMOLOGIES}

At least two twentieth-century cosmologies offered in the name of natural science would regard What caused the Big Bang? as a loaded question because it presupposes that the Big Bang really happened, and that it created our whole universe. According to Steady State Cosmology and Plasma Cosmology, there never was a cosmos-making Big Bang. As Eric Lerner, a Plasma Cosmologist, expressed it in the title of his 1991 book: The Big Bang Never Happened; but this is a small minority view among today's cosmologists. A few skeptics about the Big Bang still cling to Steady State Cosmology, ${ }^{1}$ but this position is now mainly of historical interest, except to Fred Hoyle. Lerner's challenge to Big Bang Cosmology is serious, well developed, and deserves and receives the most attention in the following pages.

\section{Steady State Cosmology}

In 1948, Big Bang Cosmology did not dominate the field as it does today. The redshift of the galaxies was well confirmed by then, and Hubble's law was available to calculate their rate of expansion. George Gamow's associates, Ralph Alpher and Robert Herman, published a paper in 1948 in which they predicted the existence of background radiation as a lasting relic of the Big Bang. They calculated its temperature at five degrees Kelvin, two degrees higher than currently accepted; but for years no one paid much attention to their prediction or tried either to confirm or falsify it. The Big Bang gained its contemporary dominance when this microwave background was actually found by radio astronomers Arno Penzias and Robert Wilson in 1965, but this information was not available in 1948.

In 1948, alternative cosmologies had a chance, especially the Steady State theory developed and published that year by Fred Hoyle, Thomas Gold, and Hermann Bondi. This theory tried to explain how the universe could appear constant, orderly, and unchanging to all observers at all times despite the fact that it is also expanding and dissipating its order and mass/energy. Steady State Cosmology said that the observable universe of all observers throughout infinite time and space would contain essentially the same quantities of entropy, background radiation, redshifted galaxies, and stars or other configurations. The heavens would always be steady or constant, on average, despite the continuous disappearance of stars and galaxies over the horizon of visibility in accord with Hubble's law. How is this possible? 
To reconcile sameness of observable mass with disappearance of observable mass, Steady State Cosmologists resorted to creation, but not the all-atonce creation of Christian or Big Bang Cosmology. Hoyle and Bondi posited an infinitely prolonged process of continuous creation. Throughout infinite space and infinite time, just enough hydrogen atoms are created to replenish the supply within every astronomer's observable universe. Given enough time, gravitational attraction forms new background radiation and new gaseous clouds from the newly created particles. Gravity concentrates these gaseous clouds into new stars and galaxies to replace those lost to view through the Hubble expansion process. Thus, throughout infinite time and space, the universe always looks basically the same, in a steady state, to all observers everywhere. ${ }^{2}$

Continuous creation is not wrought by God or any transcendent reality, according to Hoyle and Bondi. Their metaphysics was thoroughly naturalistic and this-worldly. Hoyle asked: "Where does the created material come from?" and he answered that matter is responsible for it. "Matter that already exists causes new matter to appear. Matter chases its own tail." Matter does not create new matter out of old matter or out of pre-existent energy. If it did, nothing would be left after a finite interval to feed the Hubble expansion, and the universe would be empty and dead. Like God, matter creates matter out of nothing. Just how, Steady State Cosmologists did not say.

According to Hoyle, the creation of matter by matter ex nihilo is a slow process, but in an infinite amount of time it really adds up. "The average rate of appearance of matter," he wrote, "amounts to no more than the creation of one atom in the course of about a year in a volume equal to that of a skyscraper." This is enough, nevertheless, to generate the microwave background, to cause the redshift, and to drive the Hubble expansion. "The new material produces a pressure that leads to the steady expansion," Hoyle proclaimed.

\section{Critique of Steady State Cosmology}

Most cosmologists today reject Steady State Cosmology, though Hoyle and a few others tenaciously defended it. What considerations make it implausible?

\section{A. No Observational Evidence}

Most seriously, no observational evidence supports the theory of continuous creation. Hoyle almost saw the difficulty when he conceded that "It would be quite impossible to detect such a rate of creation by direct experiment." ${ }^{.6}$ Bondi also acknowledged that "It is utterly impossible to observe directly such a rate of creation." This is certainly true. However, the problem is more serious than detecting the rate of the creation of material particles out of absolutely nothing. Namely, no instance whatsoever of such creation has ever been observed! Even if hydrogen atoms were being created ex nihilo at the rate hypothesized, no one 
could find them, no one has, and no one will. In particle accelerators, a great variety of physical particles can be created out of pre-existing particles and radiant energy; but after the initial creation of the universe itself, no matter has ever been created out of absolutely nothing. No empirical evidence at all supports the theory of continuous creation ex nihilo. Bondi, who saw this very clearly, confessed that the continuous creation of matter by matter out of nothing "is not directly observable." $\mathrm{He}$ argued for the theory nonetheless on the grounds that it is the simplest assumption compatible with the observable facts. ${ }^{9}$ Once the microwave background was discovered in 1965, this was no longer true. A few more recent Quantum Cosmologists surmise that new and enduring particles may arise from quantum fluctuations in the vacuum of empty space; but this, too, has not been verified, and there is ample room for doubt. New particles that endure are created only when actualized energy is injected from outside the vacuum..$^{10}$ The vacuum of empty space is not pure nihilo!

\section{B. The Dark Sky, Microwave Background, Redshift, and Hubble Expansion}

Without continuous creation of hydrogen atoms from nothing, Steady State Cosmology can not account for the dark sky at night, the microwave background, the redshift, or the Hubble expansion of the universe. Olbers' Paradox, according to which there should be no dark sky at night in an infinite universe, could be resolved, Bondi believed, by the supposition that redshifted light loses energy with distance-enough energy to darken most of the night sky." "Highentropy energy (in the form of radiation)," he wrote, "is constantly being lost through the operation of the Doppler shift in the expanding universe, while lowentropy energy is being supplied in the form of matter."12

Unfortunately for the theory, low entropy energy is not being supplied in the form of new matter. No fresh hydrogen atoms come into being from nothing to replace what is lost to view, to drive the redshift and Hubble expansion, to resupply the ebbing microwave background, or to explain why the sky is dark at night. The omnipresent background radiation, the almost ubiquitous redshift, Hubble's law of uniform expansion, and the dark night sky count decisively against Steady State Cosmology. No observational evidence exists that any matter is being created continuously out of nothing, much less that just the right amount of it exists to replenish what is being lost to view by a relentless Hubble expansion.

\section{The First Law of Thermodynamics}

Critics of Steady State Cosmology protested that continuous creation violates the First Law of Thermodynamics, which prohibits the creation and destruction of mass/energy. Hoyle replied that the theory is actually necessary to account for conservation only in the observable universe. Energy is conserved because 
exactly enough matter is being created to counterbalance its loss over the horizon due to the Hubble expansion. ${ }^{13}$ Bondi claimed that continuous creation "prevents the approach of the heat death, the state of thermodynamic equilibrium in which no evolution can take place and in which the passage of time has no significance."'4 These responses are inadequate.

Hoyle displaced the "Energy is constant" of the First Law of Thermodynamics, with "Energy that can be observed at any given time is constant." His position is that the total existing amount of mass/energy in the universe is constantly increasing. Real energy is being created continuously out of nothing to preserve the appearance of constancy. Both Steady State and some Big Bang Cosmologies postulate the creation of mass/energy out of nothing, either gradually, or all at once. If energy conservation is construed as a metaphysical principle that guarantees the eternal and necessary existence of all existing mass/ energy, Steady State Cosmology violates this incrementally, but in infinite quantities over infinite time. Big Bang Cosmology violates it in finite quantities during a single resplendent burst. However, as explained in Chapter One, the law of conservation is not a metaphysical principle, despite the claim of the Oscillation Cosmologies introduced in the next chapter that the energy of our universe is derived from an infinite number of prior universes. Understood scientifically, all natural laws, including the laws of thermodynamics, come into being together with the universe and do not antedate it. Steady State Cosmology violates the First Law of Thermodynamics because it posits creation of mass out of nothing within our existing universe; but Standard Big Bang Cosmology does not violate it because "prior to" the universe-creating Big Bang, nothing existed to which the law could have applied, so no such law existed.

\section{Antimatter}

In particle accelerators, matter and antimatter particles are always produced in pairs; and particle physicists believe that symmetrical particle/antiparticle production is a universal law of nature ${ }^{15}$ In the thermonuclear furnace of the Big Bang, conditions somehow permitted enough matter to prevail over antimatter to produce the universe as we know it. Within our universe, when particle/antiparticle pairs are produced, they immediately annihilate one another in detectable explosions that leave traces of gamma rays.

The law of symmetrical production obviates Steady State theory's continuous creation of hydrogen atoms. If the proton of a hydrogen atom is created, it will be accompanied by an antiproton; and if the electron of a hydrogen atom is created, a positron will accompany it. New particle/antiparticle pairs always immediately annihilate one another. Thus, endless hydrogen atom production in "empty space" is impossible. Continuous creation fails to provide for the renewal of matter and for the elimination of antimatter. If matter/antimatter obliteration were transpiring throughout space, the resulting ubiquitous explo- 
sions and the ensuing omnipresent gamma ray radiation would be readily detectable; but astronomers detect no such all-pervasive occurrences. According to Virginia Trimble, "a few things don't belong anywhere...[like] antimatter, at least not anywhere in the observable universe, or we would see gamma rays where it meets matter." ${ }^{16}$ We just don't find what the theory predicts.

\section{E. Verifying Infinity}

Philosophically, Steady State Cosmology presupposes a naturalistic metaphysics that is totally unverified and unverifiable. Hoyle explicitly affirmed the infinity of both time and space, writing that "Theory requires the galaxies to go on forever, even though we cannot see them....The galaxies are expanding out into an infinite space. There is no end to it all....The same thing applies to time."17 Bondi said that "There is no point origin and no initial catastrophe in this theory." ${ }^{8}$ Unfortunately, there neither is, nor can there be, a direct or an indirect inductive confirmation of the propositions that time extends infinitely into the past, that it will extend infinitely into the future, and that space extends endlessly in all directions. Logically, inductively, we cannot infer the infinite from the finite, which is all that we ever observe.

Is the infinity of spacetime plausible as an explanatory hypothesis? Since no empirical evidence favors it, why would anyone want to adopt this postulate? Cosmologists like Sandra Faber ${ }^{19}$ and Roger Penrose ${ }^{20}$ confess being attracted formerly to Steady State Cosmology on purely aesthetic grounds, because they found the ideas of endless time and space to be beautiful and those of an initial singularity and an absolute beginning to be ugly. In adopting a general worldview, aesthetic considerations may be inescapable in the final analysis; but most rational persons are skeptical of attempts to ground metaphysical worldviews primarily or prematurely on aesthetic preferences. Atheistic philosophers and astrophysicists definitely would not allow theologians to get away with believing in God merely because God is beautiful; so why should they get away with affirming an infinite Godless universe just because they find the thought of it beautiful?

In 1989, Fred Hoyle still defended Steady State Cosmology in an article ${ }^{21}$ and in an interview conducted in August of that year. In the interview about his earlier work, he commented: "I don't really work in terms of belief. I didn't go beyond saying that the steady theory is a possibility." ${ }^{\prime 2}$ However, his books and articles do not disclose that Hoyle cared only about possibilities. Yes, anyone who wants to know the actual world must explore possibilities, but mere possibilities are very cheap. They require no empirical knowledge at all, no experiments, and no verification whatsoever (all dear to the heart of Hoyle). As Leibniz proposed, being possible means nothing more than that the concept of something is free from logical self-contradiction. Matter that is actually antimatter is not possible; but matter that really is matter is possible. Pure possibilities 
give us no information at all about our actual world, except that it too is a possible world. Philosophers play interminable games with abstruse possibilities; but we expect more from scientific cosmologists. Usually they expect more of themselves.

To summarize, Steady State Cosmology was developed as an alternative to the somewhat primitive versions of Big Bang theory that were available in the late 1940s. Steady Staters were convinced that Big Bangers did not know what they were talking about, and vice versa. According to Hoyle and his associates, our universe is infinite in space and time; it has always been here; so no Big Bang is required to explain it. Their confidence that on a large scale the universe has always looked, on average, very much as it does today had to be reconciled somehow with the Hubble expansion. How can the observable universe always contain the same amount of intergalactic mass/energy if and when galaxies are constantly disappearing over the horizon of visibility due to the Hubble expansion process? Hoyle and Bondi resorted to continuous creation of matter out of nothing by pre-existing matter to account for the eternal renewal of mass/energy in the visible universe.

For many reasons, Steady State Cosmology is not plausible. No empirical evidence supports the continuous creation of matter out of nothing by preexisting matter. Without continuous creation, Steady State Cosmology cannot account for the background radiation, the redshift of the galaxies, the Hubble expansion, and the dark sky at night. Continuous creation of matter out of nothing violates the First Law of Thermodynamics, according to which total mass/energy in the entire universe, not just the observable universe, is constant. Particles of matter and antimatter are always created together and then annihilate one another immediately; but no observations confirm the ubiquitous and continuous annihilation that should accompany ongoing matter/antimatter creation; and if it happened, we and our material world would not be here. Finally, the boundlessness of space and time are totally unverified and unverifiable, infinitely beyond the limits of scientific methods and knowledge. These metaphysical beliefs cannot be counted as scientific knowledge, and no convincing philosophical arguments support them. Crucial evidence for Steady State Cosmology is lacking. The evidence against it is overwhelming.

Perhaps another cosmology that affirms the boundlessness of spacetime is more credible. We turn now to Plasma Cosmology. Can it succeed where Steady State Cosmology failed?

\section{Plasma Cosmology and Eric Lerner's Critique of the Big Bang}

Today, occasional minor anomalies may not quite fit, but Big Bang Cosmology is massively supported and far from discredited. Big Bang Cosmology indisputably dominates all others; but it is not without its critics. A few astrophysicists emphasize transient discrepancies between the theory and available data, but 
these discrepancies are constantly being resolved. In 1991 Eric Lerner published The Big Bang Never Happened. Lerner's primary objections to the Big Bang, the central physical and metaphysical features of his Plasma Cosmology, and the basic flaws in his position will be discussed next. Interested readers should peruse his informative and challenging book for themselves.

A few cosmologists announced during the 1980s that they had found serious difficulties with the Big Bang theory of cosmic origins. Eric Lerner builds on their work and argues that discrepancies between theory and observation are significant enough to falsify Big Bang Cosmology. As he summarizes his main criticisms,

The test of scientific theory is the correspondence of predictions and observations, and the Big Bang has flunked. It predicts that there should be no objects in the universe older than twenty billion years and larger than 150 million light-years across. There are. It predicts that the universe, on such a large scale, should be smooth and homogeneous. The universe isn't. The theory predicts that, to produce the galaxies we see around us from the tiny fluctuations evident in the microwave background, there must be a hundred times as much dark matter as visible matter. There's no evidence that there's any dark matter at all. And if there is no dark matter, the theory predicts, no galaxies will form. Yet there they are, scattered across the sky. We live in one. ${ }^{23}$

The following doubts arise about Big Bang Cosmology, according to Lerner.

\section{A. Large-Scale Structures}

Is the universe too immense for Big Bang Cosmology? Astronomers have now mapped and measured large sectors of the heavens. They found galaxies clustered together in vast strings, sheets, webs, and tapestries, separated by huge voids of seemingly empty space. These clusters of galaxies are themselves clustered into superclusters, separated by immensities of near emptiness. The superclusters are grouped into patterned megaclusters, divided by vast expanses of barrenness, almost without end. Age is correlated with size and velocity in an expanding spacetime system, and the age and magnitude of space in the observable universe are unimaginably vast.

Both the age and the size of the universe are far too great for Big Bang Cosmology, according to Eric Lerner. Big Bang Cosmology can allow for no objects older than twenty billion years at most, and no expanses greater than one hundred fifty million light years across; but the universe accessible to the best modern telescopes is radically different from what the Big Bang theory predicts. Lerner claims that some supergalaxies are between one hundred to one hundred 
and fifty billion years old, at least five times older than Big Bang theory can accommodate. ${ }^{24}$ If some galaxies formed from an earlier state in which matter was spread smoothly through space, this matter must have moved for at least eighty billion years to arrive at its visible distribution; but the Big Bang says that the entire universe is at most only twenty billion years of age. ${ }^{25}$ This theory cannot be saved by moving back the time of the initial explosion, Lerner contends, for that would be incompatible with the measured pace of the Hubble expansion. ${ }^{26}$

\section{B. Dark Matter and the Galaxies}

Does enough matter or mass/energy exist in the universe to explain how the galaxies were formed? This question is closely related to others, Lerner thinks. Is our universe closed rather than open? Does enough mass/energy exist to enable gravity to halt and reverse the universe's expansion? Enough mass to form the galaxies would equal that required to close the universe, according to Lerner. ${ }^{27}$ Other cosmologists doubt that the relation of mass to forming galaxies and closing the universe is so exact. Stephen Hawking, who concedes uncertainty about it, suggests that "A tenth of the critical density would be enough matter for galaxies and stars to form." 28

In Big Bang Cosmology, galaxies form as a result of tiny initial irregularities or fluctuational flukes in the early universe that spread over time to detach large irregular chunks of radiant energy and gases from one another. Later, many of these gaseous expanses solidified as gravity concentrated them into supergalaxies, galaxies, their stars, halos, and smaller clouds of gas and dust. Lerner insists that not enough physical mass/gravity exists in the galaxies or elsewhere to do the job. Big Bang Cosmologists assume that gravitational energy is the dominant force in the universe and that it caused the galaxies to form. Many believe that it will ultimately reverse the expansion of the universe. Without sufficient mass, there is not enough gravity for either purpose; and, Lerner contends, the observable mass definitely is not there.

The visible mass in the universe is only two percent at most of what is required to close the universe and form the galaxies, Lerner claims. Other cosmologists readily acknowledge that the luminous mass in the universe is barely one to two percent of the critical mass necessary to close the universe. ${ }^{29}$ Ninety eight percent or so of the mass required to close the universe and form the galaxies is missing, says Lerner. ${ }^{30}$ If Big Bang Cosmology is right, there should be no galaxies; but galaxies exist; so Big Bang Cosmology is wrong, Lerner concludes.

To save the theory, Lerner suggests, Big Bang Cosmologists conjure up massive quantities of cold dark matter and claim that it comprises ninety-eight percent or so of the physical universe. The trouble is, no one can find it! Astronomers can detect only the hot luminous matter that appears in the optical, ultra- 
violet, X-ray, gamma-ray, infrared, and radio regions of the spectrum; but this luminous matter falls significantly short of what Big Bang Cosmology requires. Rather than give up the Big Bang theory, its advocates concoct imaginary stuff that is not there. Big Bang Cosmology can be defended, Lerner charges, only by arbitrarily postulating the existence of purely fictional entities, a process akin to adding epicycles to defend the Ptolemaic geocentric theory of the solar system.

The cold dark matter of Big Bang Cosmology is merely deduced from theory, Lerner says, without a trace of observational support; and other prominent astrophysicists agree. ${ }^{31}$ This missing mass, theorists concede, is not conventional matter like protons, neutrons, and electrons; if it were, it would be luminous. Radically different unobserved kinds of matter must exist in massive quantities to close the universe; so theorists concoct exotic particles like heavy neutrinos, axions and WIMPs (Weakly Interacting Massive Particles) which, by their own admission, no one has ever found. Astronomer Vera Rubin mentions "neutrinos, gravitinos, photinos, sneutrinos, axions, magnetic monopoles, and dozens more." "If If they exist, they could easily supply the missing mass required to form the galaxies and close the universe. "Their only drawback," Lerner observes, is "that as in the case of cosmic strings, there is no evidence that they exist." ${ }^{33}$ This drawback, if sustained, is quite serious indeed!

Actually, we possess significant empirical evidence for the existence of some cold dark matter. It is obtained by first measuring the velocities of stars in galaxies, and of galaxies in clusters. Then the gravitational force, and thus the mass, required to hold them in orbit or cause their observed movements is calculated. Finally, the mass of detectable luminous matter is subtracted from the required mass. The results indicate that observable matter is only a small fraction of what is necessary to prevent orbiting stars and galaxies from flying apart. According to Vera Rubin, at least 90 to 99 percent of the matter in the universe is dark matter that is "detected by its gravitational attraction on the matter which we can see." ${ }^{34}$ Whether enough dark matter really exists to close the universe is a matter of heated controversy among contemporary cosmologists. As William Fowler put it, "According to the Caltech religion, the universe is open, and according to the Princeton superstition, it's closed." ${ }^{35}$

Lerner attempts to explain away all empirical evidence for dark matter. Citing research done by other astronomers, Lerner argues that dark matter is accepted by many astronomers because they greatly overestimate the masses of measured galaxies and clusters in two ways. These astronomers either count "interlopers," on this side or the other of observed galaxies as if they actually belong to the galaxies they are weighing ${ }^{36}$ or else they count small galaxies completely beyond the gravitational field of larger galaxies as if they belong to them. Either way, the mass and gravity of a measured galaxy will be greatly overestimated. ${ }^{37}$ Lerner concludes that "These two errors would account for all of the "missing mass"; in pairs of galaxies, groups of galaxies, and clusters 
there is no dark matter." ${ }^{38}$ Both of Lerner's explanations seem irrelevant, however. Whether or not interloper or nearby galaxies are beyond, before, or outside the gravitational fields of focal galaxies, their mass is there in the universe nonetheless.

Very few astronomers are persuaded that all cold dark matter can be explained away so easily. Much more evidence is available for the existence of cold dark matter than Lerner's exposition suggests. As Vera Rubin points out, the velocities of the outermost stars in spiral galaxies are not significantly less than the velocities of the innermost stars, which indicates the presence of dark matter because visible matter cannot explain this motion. Spiral galaxies are very different from our solar system, where planetary velocities decrease with increasing distance from the Sun, in accord with Newton's law that gravitational attraction decreases as distance increases. ${ }^{39}$ The luminous centers of spiral galaxies seem to contain the most matter, so we would expect the velocities of their outermost stars to decrease, like the outermost planets of our solar system; but this does not happen. ${ }^{40}$ Why? According to Rubin, "The conclusion is inescapable: matter, unlike luminosity, is not concentrated near the center of spiral galaxies. In short, the distribution of light in a galaxy is not at all a guide to the distribution of matter." ${ }^{41}$ Thus, much dark matter must be there within the galaxy and/or its surrounding "dark halo."

Lerner concludes that even if some dark matter exists, contemporary measurements of celestial mass disclose "far too little to 'close the universe' and solve the various problems confronting the Big Bang theory." 42 Many contemporary cosmologists agree. Lerner probably loses the battle over the non-existence of dark matter, but he wins the war over whether enough of it exists to close the universe. Vera Rubin's studies of galactic dynamics disclose some missing mass; but, she admits, when all this dark matter is added in, the universe still has a critical density of .2 at best, ${ }^{43}$ still far short of the critical density of 1. James Trefel remarked in $\mathbf{1 9 8 8}$ that after including all detectable dark matter, we are left with only thirty percent of the critical value required for closing the universe. ${ }^{44}$ Similarly, in $1990 \mathrm{H}$. Reeves wrote,

The best estimates of the total (baryonic and nonbaryonic) cosmic density, from dynamic effects on galactic motions, yield values around ten percent of the closure density.... There is no sound proof of the existence of a nonbaryonic matter contributing in a major way to the total density of the universe. ${ }^{45}$

In late 1995, Joshua Roth and Joel R. Primack affirmed that "counting the universe's luminous inhabitants-galaxies...only adds up to at most about one percent of the critical density," and that "galaxy halos typically contain enough dark matter to contribute at least 13 percent of the critical density, with preferred values exceeding 30 percent.." ${ }^{\prime 46}$ Many astronomers estimate the total dark 
and luminous mass to be around thirty to forty percent of critical mass ${ }^{47}$ Sixty percent or more of the mass required to close the universe can be provided only by postulating the existence of an odd attracting, not an Einsteinian repelling, Cosmological Constant, or by assigning significant mass to neutrinos. Neutrinos were found in 1998 to have a tiny mass, ${ }^{48}$ but they add only another tenth of critical mass at most; and an attracting Cosmological Constant is nothing more than an ad hoc hypothesis unsupported by empirical evidence. ${ }^{49}$ Any implausible theory can be saved if we are willing to posit enough epicycles and to violate egregiously the scientific principle of parsimony.

As far as we can tell, the density of our universe is only a small fraction of one, Omega, or critical density. Some non-luminous matter may be tied up in black holes, brown dwarfs-as confirmed by the Hubble Telescope in 1995, dim stars, and interstellar gas and dust; but astronomers were stunned in 1994 by the failure of the Hubble Telescope to find such things where most expected.

Some dark mass may be totally different in kind from any matter with which we are familiar; but when all the dark matter we can find is added to luminous matter, the total mass is at best only four percent or so of critical mass, according to an extensive review of all available evidence by Peter Coles and George Ellis in $1994 .{ }^{50}$ Recent attempts by more than one research team to "weigh" the universe by examining the redshift of distant supernovae also favor a low-density universe. ${ }^{51}$ Estimates of the grand total of dark and luminous matter obviously differ somewhat, but astrophysicists agree substantially that far too little exists to close the universe. Thus, even if some dark matter exists, this does not help very much. Emphasis on insufficient mass is not merely a Lerner eccentricity; many prominent astrophysicists agree that too little mass/ energy exists to reverse the cosmic expansion process and close the universe. ${ }^{52}$ As Coles and Ellis indicate, "On the balance of the evidence, an open Universe should be preferred." 53

Conclusive evidence that we live in an open universe now seems to be available. Astronomers were stunned again in early 1998 when two research teams arrived independently at the unexpected result that the rate of cosmic expansion is actually increasing, not decreasing, as almost everyone since Hubble assumed. Gravitation/mass is not even powerful enough to slow down the pace of cosmic expansion, as universally assumed prior to 1998, much less to stop it. Supernovae in far distant galaxies were discovered to be much dimmer and further away (by ten to fifteen percent) than predictions based on a slowing rate of cosmic expansion could explain. The best explanation of their having traveled so much further than expected is that the rate of Hubble expansion is increasing, being driven by the pervasive energy of a repelling Cosmological Constant. ${ }^{54}$ These initial findings were confirmed many times during and since 1998; and by the end of that year, the prestigious journal Science declared the increasing rate of cosmic expansion to be the "Breakthrough of the Year"! 55 
Most astrophysicists, including Stephen Hawking who at first resisted ${ }^{56}$ are now convinced that the issue is definitively resolved: we live in an open universe, one that will never end in a Big Crunch. Thus, all cosmologies that presume a closed universe are utterly implausible. Some astronomers caution that the dimmer-than-expected light from these Supernovae might be best explained by the presence of cosmic dust, or by their taking longer than usual to achieve their maximum brightness. So far, the increasing rate of Hubble expansion has withstood every challenge, ${ }^{.7}$ and even if the Hubble expansion rate is not increasing, too little mass exists in the universe to close it. High precision observations of the microwave background made in 1999-2000 seem most compatible with a flat universe having an Omega of $1,{ }^{58}$ but neither flat nor open universes ever collapse, and both kinds are absolutely incompatible with oscillationism.

We probably live in an open universe with a finite past that will expand forever, but Lerner contends that an open universe would falsify all forms of Big Bang Cosmology. Readers will soon see that and why this is not so.

\section{Cosmic Heterogeneity}

Is the universe as homogeneous and isotropic as required by Big Bang Cosmology, or is it too clumpy and structured? Without dark matter and its gravitational effects, Big Bang theory cannot account for the heterogeneity of the universe, Lerner contends; but Plasma Cosmology can readily explain the formation of stars, solar systems, galaxies, supergalaxies, and cosmic heterogeneity. Big Bang Cosmology assumes that among the four basic forces of nature, only gravity is available to consolidate gaseous regions of mass/energy into heavenly bodies or into galactic and supergalactic structures. ${ }^{59}$ Relying on the theories and research of Hannes Alfvén ${ }^{60}$ and his associates in Sweden, Lerner proposes that electromagnetism can diversify the cosmos, where gravity alone cannot; ${ }^{61}$ once we comprehend how electromagnetism operates on plasmas, we will know how the universe came to be structured.

Plasmas are "hot, electrically conducting gases;" and "Over 99 percent of the matter [in the universe] is plasma," Lerner writes. ${ }^{62}$ Plasma Cosmology says that flowing electromagnetic currents pervade our plasma universe; the entire universe is a gigantic electrical power grid: "Plasma cosmologists envision a universe crisscrossed by vast electrical currents and powerful magnetic fields, ordered by the cosmic counterpoint of electromagnetism and gravity." 63

Big Bang Cosmology ignores electromagnetism and relies on gravity alone to explain the lumpiness of the universe. This does not work, Lerner insists; but when both electricity and magnetism are considered, the heterogeneity of the universe is adequately explained. Gravity is not the only physical force that draws things together; electromagnetism does also. On a cosmic scale, it and gravity together structure the universe. Lerner's hero, Hannes Alfvén, contends 
that "Plasma becomes inhomogeneous naturally," that "Plasma, electrical currents, and magnetic fields work to concentrate matter and energy, to make the universe the complex, dynamic, and uneven place that it is." ${ }^{\prime 4}$ Plasma pervaded by electromagnetic currents naturally produces swirling filaments, whirlwinds, and vortices through a "pinch effect" that pulls currents together when they are flowing in the same direction. ${ }^{65}$ On a cosmic scale, these processes produce solar systems, stars, vast strings of galaxies, and enormous filaments of super-galaxies. ${ }^{66}$ Furthermore, "Magnetic fields and currents can concentrate matter and energy far faster and more effectively than can gravity." ${ }^{17}$ Indeed, "Plasma interactions can, given a few hundred billion years, form the supercluster complexes." This is far too long for a Big Bang universe with only twenty billion years at most to spare, but for Plasma Cosmology "time is no problem." ${ }^{18} \mathrm{~A}$ few hundred billion years hardly matter in an infinitely old universe.

\section{Homogeneity and the Microwave Background}

Does the microwave background really originate with the very earliest universe? The discovery in 1965 of the microwave background by Penzias and Wilson was crucial in persuading most cosmologists to accept the Big Bang theory of origins. Both the remarkably homogeneous black body radiation coming from everywhere in the universe, and the uniform distribution of matter on a large enough cosmic scale, seem to confirm Big Bang Cosmology's prediction of homogeneity and isotropy in the universe. Big Bangers construe the microwave background to be an enduring remnant of the age of radiation that began only minutes after the eruption of the primordial fireball. Lerner challenges the presumption that this is the only plausible explanation and offers an alternative hypothesis as much more credible.

Lerner's theory is simple. Electrons flowing through magnetic fields emit radio waves and microwaves. The microwave background originates in intergalactic magnetic fields that first absorb and then reemit microwave radiation. ${ }^{69}$ The microwave background appears to come from everywhere rather than from specific intergalactic locales because, after a number of reabsorptions and reemissions, microwaves are scattered in all directions, and their radiation is "smoothed out." 70 Thus, the microwave background originates in intergalactic space and requires no Big Bang for its explanation.

Another problem, says Lerner, is that the microwave background is too smooth. Big Bang theory proposes that fluctuations and aberrations very early in the universe eventually created supergalaxies, galaxies, and stars. If this actually happened, some inhomogeneities in the microwave background should show up, but they do not, Lerner declares. Preliminary reports from the Cosmic Background Explorer (COBE) Satellite launched by NASA in 1989 indicated that "the Microwave spectrum is 'too perfect'," and this "rules out any way of 
forming the large scale structure of the universe from the Big Bang."'1 This challenge to Big Bang cosmology is very serious, but since Lerner wrote, the tables have turned decisively against him.

\section{E. The Hubble Expansion and the Infinite Universe}

Could the universe expand in accord with Hubble's law without a Big Bang? If so, how so? Lerner's Plasma Cosmology is not easily reconciled with the Hubble expansion of the universe and the redshift from distant galaxies. Like Steady State Cosmology, Plasma Cosmology incorporates a non-empirical metaphysics of infinite space and endless time. Steady State Cosmology tried to reconcile the Hubble expansion with an infinite and eternal universe by postulating everlasting continuous creation. How can Plasma Cosmology repudiate both the Big Bang and continuous creation, yet affirm the Hubble expansion? Lerner admits that this is not easy!

The Hubble expansion cannot be explained away, though Lerner would like to do so. Most cosmologists identify the redshift of the galaxies with the Doppler effect, and Lerner agrees after examining alternative proposals. In an Appendix,${ }^{72}$ Lerner discusses and rejects two alternative explanations for the redshift, first that light simply gets tired or loses energy as it travels through long distances (as Bondi believed), next that some unknown physical law causes the scale of everything to expand with time. Lerner repudiates the first because it requires a much greater density of matter than is available and because there is no evidence that anything absorbs energy from traveling photons. The second view is unacceptable because it cannot be confirmed and because it involves new, unverifiable, and implausible laws of physics. Lerner concedes that since light arriving from a source moving away from an observer shifts toward the red end of the spectrum, the galaxies must be moving away from us as the universe expands.

To explain the Hubble expansion, Lerner prefers a proposal developed by Alfvén, according to whom it results from what I will call a "Mini-Bang," though this is not Lerner's terminology. Lerner thinks that a Mini-Bang (perhaps more than one) occurred when a limited region of the infinite universe was blown apart by a colossal matter/antimatter collision to form our observable universe.

Alfvén and Lerner are convinced that substantial quantities of antimatter exist in the infinite vastness of space. It is normally separated from our material corner of the universe by electromagnetic vortices, but occasionally matter and antimatter collide. Billions of years ago, in our small corner of the infinite cosmos, matter contracted gravitationally, not to a singularity, but to a hundred million light-years across, a tenth of its present size. ${ }^{73}$ Massive quantities of matter and antimatter just happened to be in the same vicinity, so one or more matter/antimatter explosions occurred, producing our observable expanding 
universe or "metagalaxy." If the Big Bang never happened, Lerner must still answer the question: "What caused the Mini-Bang?" He answers that ten to twenty billion years ago it resulted from a gigantic collision of matter with antimatter in our little corner of infinite space. Residual kinetic energy from that explosion accounts for the Hubble expansion, ${ }^{74}$ which was "in no way a Big Bang that created matter, space, and time. It was just a big bang, an explosion in one part of the universe."7s

Lerner's Plasma Cosmology thus resorts to a Bang after all, but only a metagalaxy-producing Mini-Bang. Not surprisingly, he abhors the outcome that he embraces. ${ }^{76} \mathrm{He}$ concludes, "The question of the Hubble relation remains unanswered," and "Far more theoretical and observational work is needed." But "Why the Hubble expansion?" remains unanswered only if Big Bang Cosmology is rejected! Big Bang theory provides a very plausible answer!

Plasma Cosmology invites a number of serious questions. Is the entire infinite universe expanding like the observable universe? Is this a material world through and through? Plasma and Big Bang Cosmologies give very different answers.

What would Plasma Cosmologists expect to find if we could see very deeply into infinite spacetime? What are those parts of the universe like that were not affected by our localized Mini-Bang? Lerner does not answer, but we can make some educated guesses and predictions. Since most of the allegedly infinite universe was not affected by our Mini-Bang, it would not be involved in our Hubble expansion. Other metagalaxies might express their own expansions or contractions. Some of them might be headed directly toward us. Some might be composed of antimatter or, heaven forbid, even dark matter; and they could exhibit radically different natural laws.

How could our own metagalaxy belong to the "same universe" with innumerable causally unrelated metagalaxies? Lerner neither asks nor answers, but the question deserves some serious consideration. Could any two metagalaxies belong to a single universe without any causal connections or lawful spatiotemporal continuities, at least at their edges? These edges might be so far away from local astronomers that they could not observe any metagalactic interactions. Totally independent universes might exist without causal contact and spatiotemporal continuity; but if metagalaxies belong to the same universe, surely they must affect one another, be spatiotemporally continuous, and share many if not all natural laws. Actually, Plasma Cosmologists do not have the slightest trace of scientific evidence that the universe is infinite, that other metagalaxies exist (like Lerner's alleged matter and antimatter worlds that collided to produce our world), or that they are distantly continuous with our own. Even if these presumptions were true, we could never know it. Very few, if any, metagalaxies in Lerner's infinite universe ever affect our observable world, so how could they belong to our universe? 
Big Bang cosmologists, by contrast, do not identify the whole expanding universe with the observed or observable universe, but they think that it is finite and in principle observable and continuous with our own causally, spatially, temporally, and in other ways. They expect to investigate more and more of it as better instruments like the Hubble Space Telescope are deployed, repaired, and upgraded. They predict that the most general features of the as yet unseen far distant universe will significantly resemble what we have seen already. This has always been the case as better scientific instruments have allowed us to probe deeper and deeper into the unknown universe, and it should not change. Big Bang Cosmologists expect the now unknown universe out there to be composed mostly of matter, not antimatter, and to obey familiar physical laws, including Hubble's law of uniform expansion. Results obtained from the Hubble Space Telescope consistently confirm these expectations.

The further out we look in space, the further back we see in time; even at the speed of light, considerable time, billions of years in some instances, lapses before photons and radiant energy reach us from far distant objects. Big Bang Cosmologists expect the most distant objects in the universe to be moving away from us and from one another at speeds roughly proportional to their distance, with minor variances due to local gravitational fields. These speeds, they anticipate, will gradually approach the speed of light. They expect that the most distant objects in the heavens will be observed in their youth, as they were billions of years ago when their light and radiant energy now arriving here departed from there. So far, with every improvement in technology, their predictions pan out.

Some of the most distant objects that astronomers have discovered thus far are the quasars-starlike objects that contain as much mass/energy as entire galaxies. In late 1998, quasars that are thirteen billion light years away were detected. ${ }^{78}$ Quasars, seen in their youth, are some of the most distant and thus the oldest celestial objects known to us, but they obey familiar physical laws. In January 1993, radio astronomers, using the facilities at Kitt Peak Observatory in Arizona, discovered giant clouds of gas twelve billion years old in which galaxies are being formed. These clouds contain as much mass as entire galaxies, but no stars had formed in them twelve billion years ago. Since 1996, many additional observations with the Hubble Space Telescope disclosed galaxies as they existed nearly twelve billion years ago, within three billion years of the Big Bang, and found them to be smaller but much closer together and more numerous than those nearer to us in space and time; the small early ones probably merged over time to form the large later galaxies. ${ }^{79}$

The Hubble Space Telescope now provides us with a vision of embryonic protostars and new stars being formed in and ejected from dense clouds of space dust ${ }^{80}$ Big Bang theory predicts that galaxies go through gaseous phases before they develop into stars and galaxies; and this prediction is now confirmed. In 1994, in a closer galaxy, astronomers identified for the first time a planet in 
orbit around a distant sun in our Milky Way. By the end of 1995 at least four were recognized, with new ones being added almost every month thereafter. By the end of 2000, nearly fifty or so were known, some earth-size, most Jupitersize. ${ }^{81}$ As we learn more and more about our universe, we find no far distant metagalaxies or alternate antimatter universes. We just find more and more of what Big Bang Cosmology predicts.

Nothing can be accelerated through existing space faster than the speed of light and cross the speed of light barrier, says the theory of relativity; but the Hubble expansion of space itself can separate celestial objects at faster-thanlight speeds, thereby making it impossible for these objects ever to communicate causally. This is the "horizon problem" discussed earlier. According to Hubble's Law, each time the distance doubles, the speed doubles. Without violating relativity, some galaxies are moving away from local observers at speeds that exceed 186,000 miles per second, the approximate speed of light. Objects moving away from us faster than light are forever beyond our horizon of visibility. Their light will never reach us. This is one good reason why we cannot identify the observable universe with the total universe that originated with the Big Bang. Though his estimate is admittedly only approximate, Alan H. Guth surmises that "...The entire universe is expected to be at least $10^{23}$ times larger than the observed universe;...the observed universe is only a minute speck in a universe that is many orders of magnitude larger." ${ }^{82}$ This largeness should not be confused with infinity, however.

Astronomers look back in time as they see further away in space, but Big Bang Cosmologists do not expect to see the Big Bang itself, or anything beyond and before the Big Bang. No optical telescope will ever see through the fog of cosmic radiation that lasted for the first 700,000 years. Since the microwave background dates back to the onset of this age of radiation around 300,000 years after the Bang, radioscopes already see at least that far back in time. Milton Munitz speculates that if we could develop telescopic instruments capable of detecting neutrinos, gravitons, magnetic monopoles, or free relic quarks, we could access information coming from the earliest fraction of a second after the Bang. ${ }^{83}$ Unfortunately, at present we are far from having such instruments. Commenting on the Ligo and Lisa observatories now being constructed to detect gravity waves, Gary H. Sanders and David Beckett observe,

As early as $10^{-43}$ second after the Big Bang, space and time became differentiated and gravitational radiation was able to stream freely in all directions. In principle, LIGO could detect these primordial waves, enabling us to hear at last the hush of the universe's birth. ${ }^{84}$

From this also we are infinitely far removed in practice. We do not know much about our universe, but everything that we do know favors Big Bang Cosmology. 
To summarize, Eric Lerner avidly repudiates the Big Bang theory of cosmic origins. He contends that the Big Bang's ten to twenty billion year age for the universe is far too small to accommodate recently mapped supergalaxies that are a hundred and fifty billion years old. Without massive quantities of dark matter, there is too little gravity for the Big Bang cosmos to form stars, galaxies, and supergalaxies; and no good evidence indicates that any cold dark matter exists. Big Bang theory is oblivious to the power of electromagnetism to form heterogeneous astronomical structures. Electromagnetism accounts for the microwave background without a Big Bang, and the COBE satellite found no evidence of galaxy-producing fluctuations in the cosmic background radiation.

Defenders of the Big Bang can offer intelligent responses to these doubts.

\section{Critique of Plasma Cosmology}

Plasma Cosmology has its own grave defects, some concerning its physics, and some its metaphysics.

Lerner's contention that the universe is infinite in space and time sounds remarkably like old fashioned naturalistic metaphysics. Is he a Naturalist? Many Theists, he notes, also believe that the universe is unbounded in space and time, and they regard an infinite world as an expression of God's infinite creative power. Merely believing in infinite spacetime does not make one a Naturalist. Other Theists believe that the universe is finite in time and space, even though a finite universe implies no more than finite Divine power and creativity. ${ }^{85}$

Lerner avows that Plasma Cosmology is neutral with respect to atheism and theism, that it "does not demand a creative God and is perfectly compatible without one. But nor does it preclude a creative deity." ${ }^{86}$ This cunning evasion does not successfully conceal Lerner's commitment to a Humanistic Naturalistic worldview. He contends that scientific method alone yields truth, and he rejects all "mythological" explanations. ${ }^{87} \mathrm{He}$ develops an optimistic, futuristic, and humanistic understanding of mankind and human values ${ }^{88} \mathrm{He}$ makes no appeal to God, and twice he expresses a decided preference for a naturalistic theory of origins. ${ }^{89} \mathrm{He}$ vehemently defends the principle that all events have (natural) causes against what he regards as irrationalism, occultism, mythology, and mysticism. He even finds antiscientific perspectives, which he opposes, in fashionable but fanciful metaphysical interpretations of quantum physics. ${ }^{90}$

But Lerner's naturalistic Plasma Cosmology has its own serious problems.

\section{A. A Universe Infinite in Space and Time}

Unlike Big Bang Cosmology, Plasma Cosmology affirms that the universe is infinite in both space and time. Is it reasonable, is it scientific, to adopt these features of Lerner's Naturalism? If scientific method alone yields truth, what scientific evidence proves that we live in an infinite universe? None at all, even 
if we do! Early in his book, Lerner proclaims that "Philosophers such as Nicholas of Cusa and Giordano Bruno had advocated the idea of a universe unlimited in time and space, eternal and without beginning. But no scientist had justified these notions with hard data." ${ }^{91}$ These words tacitly promise to provide hard data later to prove that space and time are infinite; but Lerner never fulfills this promise. Later he reaffirms that the finitude or infinitude of the universe "is a scientific question that must be answered by observation;" but almost immediately he concedes in a footnote that "Strictly speaking, it is not possible to prove scientifically that the universe is infinite. But it is quite possible to claim that we have no observational evidence that it is finite." ${ }^{92}$ Thus, he argues, lack of evidence for finitude counts as evidence for infinity. Are his claims plausible?

First, we observe only finitude, so all our observations count only for finitude. More importantly, we really cannot show that the universe is infinite merely because we have no evidence for its finitude, or against its infinitude. All appeals to lack of evidence are fallacious arguments from ignorance, and they establish nothing.

The naturalist Sidney Hook wrote,

The existence of God, immortality, disembodied souls or spirits, cosmic purpose or design, as these have been interpreted by the great institutional religions, are denied by naturalists for the same generic reasons that they deny the existence of fairies, elves, leprechauns, and an invisible satellite revolving between the earth and the moon. ${ }^{93}$

So, why should we disbelieve in the existence of such fanciful entities? We could argue that God, disembodied souls, cosmic purpose, pixies, elves, and invisible satellites between us and the moon really exist just because there is no observational evidence against them. No one with any knowledge of logic would accept such an argument from ignorance. Yet, Lerner's central argument for the infinity of nature is no better. Neither logic nor scientific method entitle us to believe something simply because no evidence disproves it. We would surely have to accept the reality of every occult entity imaginable if having no observational evidence against their existence counts decisively as evidence in their favor. Yet, this is all that Lerner or any other naturalist can say for their infinite and self-sufficient universe-there is no evidence against it (which ignores the Big Bang). Lerner's Naturalistic metaphysics hangs on an argumentum ad ignorantium.

We really should disbelieve in pixies, elves, and invisible satellites because, after a thorough search, we find no observational evidence for their existence. Their existence is excluded by the presence of other things that we know to exist. Thorough searches yield knowledge, not ignorance, and justify many negative conclusions. Negative propositions like "There is no butter in the refrigerator," "Unicorns don't exist," and "No stars are composed of antimatter" 
can be confirmed by observation to a very high degree of probability. If we are in no position to make a thorough search, we may not know some things for certain; but we can still know many negative truths within limits of high probability. Still, complete lack of evidence for one theory does not establish its opposite.

So what does the available evidence actually show about our universe? Our most thorough searches disclose only finitude. Empirically or scientifically we can only know finitude, even if the universe is actually infinite. Further, all the positive empirical evidence for the Big Bang (given in Chapter One) counts decisively against the infinity of space and time. Many allegedly scientific cosmologies covered in later chapters accept the Big Bang; but they postulate other universes in ways that leave all accessible evidence and all natural science far behind. For them and for Plasma Cosmology this is a great weakness.

Another subtle but unsound argument for infinite space and time runs through Lerner's book, one from historical association. He says that historical figures like Anaxagoras, Nicholas of Cusa, and Giordano Bruno, who captured the spirit of science and scientific method also believed in an infinite universe. He insinuates that these historical facts somehow imply that the universe really is infinite; but this does not follow. How did Lerner's historical heroes know that the world is infinite? What empirical evidence for it did they adduce? None!

The truth about how Naturalists like Lerner arrive at an infinite universe surfaces when he writes, "Plasma cosmology assumes that, because we now see an evolving, changing universe, the universe has always existed and always evolved, and will exist and evolve for an infinite time to come." here is "assumes"; but his opponents can just as easily assume the contrary, if that is all that there is to it. Lerner's Infinite-world Metaphysics is merely an unjustified assumption, merely an expression of groundless metaphysical faith. No logic warrants reasoning from finite observational premises to conclusions that affirm infinity, and all the positive evidence for the Big Bang weighs heavily against Lerner's position.

\section{B. Hydrogen and An Infinitely Old Universe}

Plasma Cosmology confronts scientific as well as metaphysical obstacles. Our universe is around seventy five percent hydrogen, as spectrographic scans of the heavens repeatedly confirm. If the universe were infinitely old, as Plasma Cosmology maintains, no hydrogen would exist in it today, given the laws of physics as we know them. Hydrogen is constantly being synthesized into helium and heavier elements in the stars; and this hydrogen cycle is irreversible. If nucleosynthesis of hydrogen into helium and the heavy elements has occurred throughout infinite space for an infinite amount of time, every hydrogen atom in the infinite universe would have passed through an infinite number of explod- 
ing supernovas, each of which would have converted some hydrogen into heavier elements. If this has been transpiring for an infinite amount of time, no hydrogen would remain in the universe today. Yet, the hydrogen is there. It makes up nearly seventy five percent of the universe as we know it, and its existence is overwhelming evidence against an infinitely old universe.

Fred Hoyle raised the foregoing objection against his own Steady State Cosmology. ${ }^{95}$ To avoid the difficulty, he resorted to the implausible continuous creation of hydrogen atoms. Can Plasma Cosmology provide an alternative hydrogen-renewing mechanism? Electromagnetism cannot save the theory, for it does not reverse the process of nucleosynthesis. Could Lerner's Mini-Bang solve the problem for Plasma Cosmology? Can hydrogen be renewed periodically in the infinite universe by matter/antimatter annihilations that create infinitely many Hubble-expanding metagalaxies like our own? Lerner has not worked out the physics for this; and the physics that we know is decidedly against it. Matter/antimatter explosions always produce gamma rays, never hydrogen or helium atoms, the dominant elements in our universe.

Besides, no convincing evidence indicates that antimatter exists in sufficient quantities to produce metagalactic Mini-Bangs. ${ }^{96}$ Lerner concedes that evidence for the existence of massive quantities of antimatter is inadequate, and he pleads for "more observation." ${ }^{97}$ Plasma Cosmology replaces the open universe's enigma of the cold dark matter that is not there with that of the antimatter that is not there!

Observation actually shows that very little antimatter exists in the universe, as far as we can tell. If some far away galaxies are composed of antimatter, it might seem difficult at first to know this, according to I. L. Rozental, because "Antimatter emits photons in absolutely the same way as matter does." 98 To us, light from antimatter galaxies would look exactly like light from galaxies composed of matter. However, Rozental adds, galaxies emit particles as well as photons; and antimatter galaxies would emit antiprotons and positrons. If vast quantities of each exist, then somewhere in space antiparticles would be colliding constantly with material protons and electrons. Significant and detectable matter/antimatter annihilations would result; but none have been discovered after extensive searches; so the existence of antimatter galaxies is extremely doubtful. ${ }^{99}$

Lerner might reply that although no discernible antimatter exists now, it might still have existed at the time of the Mini-Bang. According to the Big Bang theory itself, the initial universe-creating thermonuclear explosion was driven primarily by stupendous matter/antimatter collisions; so it too must reconcile the past existence of antimatter with its virtual non-existence at present.

Which theory offers the most plausible account of the origin and presence of the antimatter that fueled its Bang? In Big Bang theory, either a surplus of matter prevailed from the outset as a given initial condition of creation, or else both antimatter and matter emerged from $\mathrm{X}$ bosons (derived, in turn, from pure 
energy during the first few pre-matter minutes of creation) that decayed irregularly into a material world. How does Plasma Cosmology explain the origin of the massive quantity of antimatter involved in its Mini-Bang? How was it separated from matter? How did it survive annihilation by matter prior to its collision with a material world undergoing metagalactic gravitational collapse?

Lerner recognizes that antimatter is produced on earth whenever matter is derived from energy, so it would be an immense puzzle, he suggests, if this did not happen throughout the universe. ${ }^{100}$ Yet, whenever this happens on earth, matter and antimatter immediately annihilate one another, so it would also be a great enigma if this too does not happen throughout the universe; but it does$n$ 't. Lerner claims that antimatter can be separated from matter if both pass through a magnetic field containing a current that squirts matter out in one direction and antimatter out the opposite way. Clouds of matter and antimatter would then form and be pushed apart by the Hubble expansion. Multiple metagalactic gravitational collapses might later bring them together again to produce a Mini-Bang. ${ }^{101}$

This explanation cannot be verified directly, Lerner admits. ${ }^{102}$ Neither can the standard Big Bang explanation of why we live in a material world, we must concede. The true test of a scientific theory, Lerner contends, is the correspondence of its predictions with observation. Apparently, he regards his cosmology as an explanatory hypothesis that is justified by its fruits; but Plasma Cosmology's explanation of why we do not live in an antimatter world flunks this test. Big Bang Cosmology, with all the supporting evidence presented in Chapter One, passes.

\section{The Vastness of the Universe}

Lerner proclaims that the visible universe is too vast to have been produced within Big Bang's paltry ten to twenty billion year-old universe. Recall his claim that astronomers have now mapped supergalaxies that are at least one hundred to one hundred and fifty billion years old. This would make them at least five times older than the Big Bang's entire universe. Something has to give!

In astronomy, ages are related to velocities, distances, magnitudes, and masses; and our estimates of age depend on what we know about such things. Lerner's predicament may appear at first to arise because estimates of galactic and supergalactic ages, velocities, distances, sizes, and masses are notoriously inexact. Methods available to astronomers do not yield very precise measurements; but improved methods, enhanced by the power of the Hubble Space Telescope and the Hipparcos Satellite have already given us a much more accurate perspective on the age and composition of the stars and of the cosmos. Astronomical distances are judged by such methods as the parallax, which indicates the displacement of celestial objects when observed at the same 
moment by two different observers in two different places, by the intrinsic brightness of certain stars like the Cepheid variables that function as standard candles, and by spectral analyses of redshifts. ${ }^{103}$ The Hubble Space Telescope now views galaxies so far removed from us that Cepheids are not detectable; so today's astronomers are using the intrinsic brightness of exploding supernovae as standard candles for measuring vast cosmic distances.

Hubble's law of uniform expansion affirms that celestial distances increase uniformly in proportion to velocity, and the Hubble constant sets the scale of the universe as the ratio of distances to velocities. Much more detailed and exact measurements of cosmic distances and ages made by the repaired and upgraded Hubble Telescope and by the Hipparcos Satellite ${ }^{104}$ have already produced more evident and precise estimates of the age of the stars and of the universe; and it will continue to do so.

Still, the inexactness of measurement on an astronomical scale does not procure more than an eight to twenty billion year variance for the age of the universe. It fails to supply the hundreds of billions of years required by Lerner's "ancient structures." Lerner does not say where he gets his figures. He seems to do his own calculations, but most astronomers simply do not accept them. Discrepancies between dates and data may be dismissed as only a "temporary difficulty," but not a "permanent breakdown of the Big Bang itself." 105 In 1993, George Smoot concluded from his research team's momentous study of the cosmic background radiation by the COBE satellite that doubters about the Big Bang are now proved wrong because

The existence of the wrinkles in time as we see them tell us that big bang theory, incorporating the effect of gravity, can explain not only the early formation of galaxies but also the aggregation within 15 billion years of the massive structures we know to be present in today's universe. This is a triumph for theory and observation. ${ }^{106}$

Today, most astronomers would agree, not without justification, that Lerner and others of like mind plainly miscalculate the age and the vastness of the most ancient structures in the visible universe. The best available numbers clearly support the "Standard Big Bang" model of the origin of the universe. ${ }^{107}$

\section{Mini-Bangs and the Age of the Universe}

If the visible universe is really too vast to have been produced in a mere ten to twenty billion years, as Lerner maintains, then neither Big Bang nor Plasma Cosmology can accommodate the discrepancy. Plasma Cosmology resorts to one or more Mini-Bangs to explain the Hubble expansion, so it has exactly the same problem. If the Big Bang cannot account for the age and expanse of the visible universe, how could Plasma Cosmology's Mini-Bang do any better? In 
describing what followed the Mini-Bang, or the series of them, that allegedly produced our visible world, Lerner fumbles to avoid this difficulty.

Trapped in magnetic fields, these particles drove the plasma apart over hundreds of millions of years. The explosions were small enough not to disrupt previously formed filaments of plasma, so these far more ancient objects still exist today, in expanded form-just as designs printed on a balloon persist while it is inflated. ${ }^{108}$

Exactly what Lerner is getting at here is obscure. Are his "ancient objects" within the visible universe, or do they exist only in some theoretically constructed but unexperienced universe? Assuming the first, Lerner may have something like this in mind: The most distant parts of the visible universe contain galaxies and supergalaxies that are a hundred to a hundred and fifty billion years old, he claims. These did not participate in and were not affected by the Mini-Bangs that created the Hubble expansion. Thus, one hundred and fifty billion-year-old structures can exist within a metagalaxy that is only ten to twenty billion years old. The force of the Mini-Bangs that created our visible universe bypassed these structures, but somehow they were swept into our visible universe. How did this happen? Why did the Mini-Bangs not destroy them?

Because vast cosmic structures that antedate our Mini-Bang(s) (which other astronomers cannot find) would defy Hubble's law of uniform expansion, Lerner's explanation does not work. Except for gravity-bound galaxies like the Andromeda Galaxy and our own Milky Way, all structures in the visible universe, no matter how vast, participate uniformly in the Hubble expansion on a cosmic scale. None proceed through the heavens at a pace that drastically defies Hubble's law. Beyond gravitationally bound local groups, all galactic and supergalactic structures take part uniformly in the Hubble process. According to Sky and Telescope, "that hundreds of galaxies, including our own, are collectively swarming toward a super-massive entity dubbed the Great Attractor...describe(s) a subtle distortion in the universe's otherwise stately expansion, not its wholesale reversal." ${ }^{109}$ Only a single Bang with cosmic-wide effects can account for such ubiquitous cosmic homogeneity. No vast visible plasma filaments exist that are exempt from the effects of a single initial blast.

Lerner concedes that his "ancient objects" exist today "in expanded form"; but what caused their expansion if they were not brought into being, affected by, or disrupted by his own initial Mini-Bang? A matter-antimatter explosion powerful enough to initiate our expanding observable universe would either destroy all nearby pre-existing structures or push them forever beyond our horizon of visibility. Lerner himself cannot account for structures that are hundreds of billions of years old within a metagalaxy he concedes to be only ten to twenty billion years of age. 


\section{E. Electromagnetism and the Age of the Universe}

Gravity is too weak, Lerner insists, to concentrate mass/energy into galaxies and supergalaxies in only ten to twenty billion years. However, if, as Lerner maintains, electromagnetic forces can concentrate matter and energy much more effectively and rapidly than gravity, and both gravity and electromagnetism are available to concentrate mass, then his own plasma physics should help to explain how the stars, nebulae, and large scale structures of the universe came into being within Big Bang time. Plasma physics itself seems to make Plasma Cosmology's infinite and eternal universe unnecessary and comes to the support of the Big Bang. Calculations involving causal processes that can concentrate mass into galactic structures and help close the universe must encompass both electromagnetic and gravitational attraction, if Lerner is right. Perhaps electromagnetism and gravity conjointly determine large-scale cosmic structures; and together they determine whether our universe is open-to expand forever, or closed-to slip some day into reverse. Astrophysicists must do the computations; but the recent discovery that the rate of Hubble expansion is increasing, not decreasing, clearly indicates that the combined force of gravity and electromagnetic pinching is insufficient to reverse the course of cosmic enlargement.

\section{F. Background Radiation}

Lerner holds that the microwave background can be explained adequately without any appeal to the Big Bang. If he is right, his own metagalaxy-creating Mini-Bang would produce background radiation equal to that produced by a cosmos-creating Big Bang. Yet, all observable background radiation, he maintains, was produced by intergalactic electromagnetic fields. He takes no notice of all this extra background radiation. How much background radiation should astronomers expect to find if Plasma Cosmology is correct? If the radiation Lerner believes to be produced by intergalactic electromagnetic fields is added to the radiation residue of his Mini-Bang, the total would far exceed all the measurable background radiation in the universe. One Bang, whether Mini or Maxi, accounts quite sufficiently for all detected cosmic background radiation. Lerner's Mini-Bang is incompatible with his electrodynamic solution to the background radiation problem. If intergalactic electromagnetic fields could generate all observable background radiation, and if his Mini-bang would do exactly the same, there should be twice as much background radiation as there actually is. But there isn't.

\section{G. The Smoothness of the Universe}

With new instruments of exploration like the Cosmic Background Explorer (COBE) Satellite, astronomers can now "see" back to within 300,000 years after 
the Big Bang. In the preliminary COBE report cited by Lerner, the universe appeared to be too smooth to support the Big Bang account of the origin of heterogeneous structures in the universe like supergalaxies, galaxies, and stars. Originally, as Lerner suggests, COBE investigators found no evidence of fluctuations in the microwave background radiation. ${ }^{110}$ After he published The Big Bang Never Happened in 1991, another more definitive report on COBE data based on much more detailed analysis was presented to the American Physical Society meeting in Washington, D. C. on 23 April 1992 by George Smoot, who headed the team of COBE researchers. ${ }^{11}$ Members of the Society were electrified by the announcement that "ripples" were indeed detected in the cosmic background radiation.

Variations in temperature thirty millionths of a degree warmer or cooler than average were discovered on a scale of five hundred million light years across. These were originally very tiny, probably no greater than quantum level fluctuations; but over time minute differences become vastly expanded. The astrophysicist Michael Turner remarked that "The Holy Grail has been found. It's that important. If this evidence holds up to scrutiny, it is what we've been looking for 20 years. It confirms our ideas of how structures form." 12 Stephen Hawking, exaggerating a bit, called the COBE findings "the discovery of the century, if not of all time." ${ }^{13}$ COBE Satellite data also provided evidence for the existence of dark matter, though still far from enough to close the universe. Thus, a primary source of information about the cosmos cited by Lerner to support his position now counts decisively against it.

To summarize, like Steady State Cosmology, Plasma Cosmology affirms the infinity of space and time. Naturalistic atheists like Lerner assume that an infinite universe has always been here, is totally self-sufficient, and requires no Big Bang and no God for its creation. Yet, empirical knowledge reaches its limits long before we arrive at spatiotemporal infinity, so Lerner's avowedly scientific metaphysics is scientifically baseless.

Lerner tries to refute Big Bang Cosmology and offers Plasma Cosmology in its place. He emphasizes the dominance of gaseous plasmas in cosmic structures and the enormous cosmic effects of electromagnetism; but Plasma Cosmology comes to grief over the redshift and the Hubble expansion. Lerner reluctantly postulates a Mini-Bang resulting from the gravitational collapse and collision of antecedently existing regions of matter and antimatter to explain the creation of our observable expanding metagalaxy. He affirms that the universe beyond our metagalaxy is infinite and implies that it is not involved in our Hubble expansion. He cannot confirm this, and we should doubt it for many good reasons. If the universe is infinitely old, no hydrogen would remain in it today; but it is almost seventy-five percent hydrogen. Plasma Cosmology offers no mechanism to reverse the process of nucleosynthesis of hydrogen into heavier elements in the stars. No matter-antimatter Mini-Bang could reverse the hydrogen cycle. 
Lerner's visible universe was itself supposedly caused by a Bang ten to twenty billion years ago, so his own theory has the same (unsolved?) problems of scale that he attributes to the Big Bang. If some structures in the visible universe really are a hundred and fifty billion years old but nevertheless participate uniformly in the Hubble expansion, Lerner's own twenty billion-year-old visible universe cannot accommodate them. Most cosmologists do not accept the exaggerated age and size that he assigns to anomalous cosmic structures. If electromagnetism concentrates energy more rapidly than or in addition to gravity, this mechanism actually supports the contention that all visible structures in the universe came into being within Big Bang time.

Lerner's Mini-Bang would also produce a microwave background, so there would be far too much cosmic radiation if, as he contends, intergalactic electromagnetism generates all discernible background radiation. The latest results from the COBE Satellite actually confirm the reality of diversifying structural fluctuations in the microwave background. They also confirm that our universe is exceptionally fine-tuned for life. Calculations by Max Tegmark and Martin J. Rees "show that if the CMB's (Cosmic Microwave Background's) temperature variations were as little as ten times greater or smaller, life as we know it would not exist today." 114

Lerner cannot establish an infinite spatiotemporal metaphysical background for his Mini-Bang. On the whole, his case against the Big Bang is very weak. The Big Bang theory of cosmic origins is still without a serious rival. But how did the Big Bang itself originate? What caused the Big Bang? At this point, we still have no plausible answer; but other possibilities remain to be explored. 